\title{
COSECHA DE CASHAVARA Desmoncus polyacanthos MARTIUS, EN INUNDABLES DE LA AMAZONIA PERUANA
}

\section{Gustavo TORRES VASQUEZ ${ }^{1}$, Evelin RODRIGUEZ REATEGUI ${ }^{2}$, Cesar DELGADO VASQUEZ ${ }^{3}$, Dennis DEL CASTILLO TORRES ${ }^{1}$}

1 Instituto de Investigación de la Amazonia Peruana (IIAP), Programa de Investigación en Manejo Integral del Bosque y Servicios Ambientales (PROBOSQUE). Iquitos, Perú, gtorres@iiap.org.pe

2 Universidad Nacional de la Amazonia Peruana (UNAP), Facultad de Ciencias Forestales, Iquitos, Perú, evelita_20@hotmail.com

3 Instituto de Investigación de la Amazonia Peruana (IIAP), Programa de Investigación en Biodiversidad Amazónica (PIBA), Iquitos, Perú.

\section{RESUMEN}

Polyacantha Desmoncus es una palmera trepadora que se ocurre en las zonas bajas de la Amazonía peruana, donde se le conoce como cashavara o varacasha. La especie es utilizada por la población rural en las artesanías y la fabricación de muebles. Parcelas de evaluación se establecieron alrededor de los lagos Supay y vainilla (distrito de Jenaro Herrera) para evaluar la aparición y crecimiento de estipes, la generación de nuevos brotes, y la salud y el vigor de las plantas maduras. El experimento constó de cuatro tratamientos (intensidades de cosecha), las cuales se evaluaron durante diez meses. Los resultados muestran grandes diferencias entre los tratamientos, especialmente en el crecimiento longitudinal de estipes de cashavara, destacando claramente el tratamiento tres (T3: longitud promedio $883.66 \mathrm{~cm}$ ), con un valor promedio de $883,66 \mathrm{~cm}$. El menor crecimiento longitudinal de estipes se reporto para el tratamiento (T1: longitud promedio de 282,18 cm). Las evaluaciones de vigor de la planta (tallos) de D.polyacantha, mostraron buen vigor en el $90,60 \%$ de las plantas, vigor normal en $6.3 \%$, y sólo el 3,10\% de mortalidad.

PALABRAS CLAVE: Desmoncus polyacanthos, cashavara, estípites, bosques inundables.

\section{CASHAVARA HARVEST Desmoncus polyacanthos MARTIUS IN THE FLOOD FORESTS OF THE PERUVIAN AMAZON}

\begin{abstract}
Desmoncus polyacantha palm climber that occurs in the lowland areas of the Peruvian Amazon, where it is known as cashavara or varacasha. The species is used by rural people in handicrafts and furniture-making. Evaluation plots were established around lakes Supay and Vanilla (Jenaro Herrera district) to evaluate the appearance and growth of stipes, generation of new shoots, and the health and vigor of mature plants. The experiment included four treatments (harvest intensities), which were evaluated over ten months. The results show large differences among treatments, especially in longitudinal growth of cashavara stipes, clearly highlighting treatment three (T3: average length $883.66 \mathrm{~cm}$ ), with an average value of $883.66 \mathrm{~cm}$. The lowest longitudinal growth of stipes was reported for treatment (T1: average length of $282.18 \mathrm{~cm}$ ). Evaluations of plant strength (tillers) from $D$. polyacantha, showed good vigor in $90.60 \%$ of plants, normal vigor in $6.3 \%$, and only $3.10 \%$ mortality.
\end{abstract}

KEYWORDS: Desmoncus polyacanthos, cashavara, stipes, floodplain forest 


\section{INTRODUCCIÓN}

Desmoncus es el único género de palmeras trepadoras en América tropical. Se caracteriza por formar una mata (macolla) no muy densa, cuyos tallos flexibles alcanzan las copas del estrato medio del bosque, apoyándose para ello, en los árboles contiguos, poseen finas espinas de hasta $6 \mathrm{~cm}$ de largo que cubren regularmente los tallos. Para treparse sobre los individuos sostenes esta especie utiliza sus hojas, cuyo ráquis termina en un apéndice en forma de garfios (Pineda et al., 1995; Chinchilla, 1993; Henderson, 1995). Este genero de palmera debido a sus características mecánicas (flexión y tracción) puede utilizarse como alternativa en el reemplazo del ratán Calamus sp. (Muños \& Carpio, 1995).

En la Amazonía Peruana predomina la especie Desmoncus polyacanthos, localmente conocido como "cashavara" o "varacasha" por la cantidad de espinas presentes en toda la superficie de la planta. Habitualmente esta especie se encuentra en los bosques inundables de la Amazonía Peruana, siendo afectado periódicamente por las aguas de los ríos durante la época de inundación, pero lográndose recuperar rápidamente (Torres et al., 2010).

Las fibras obtenidas son producto de la cosecha de los estípites maduros y son destinados al mercado local para su utilización en artesanías o cestería. En las ciudades mayores son usadas en la industria de muebles principalmente como complemento de los espaldares y asientos conocidos como "esterillados". La extracción artesanal consiste en cortar el estípite en la base y jalarlo hasta dejarlo caer al suelo. Luego mediante una clasificación se separa la parte aprovechable (desde la base hasta la parte madura), posteriormente se quita la corteza exterior conjuntamente con las espinas que se encuentran en el estípite (Figura 1). Este proceso de extracción tradicional no tiene en cuenta la clasificación de los estípites presentes en la mata, tampoco alternativas de manejo de los estípites remanentes. En este sentido el presente estudio tuvo como objetivo generar información sobre técnicas de cosecha sostenible para el manejo de cashavara en la Amazonía Peruana y de esta forma contribuir a la conservación de la especie.

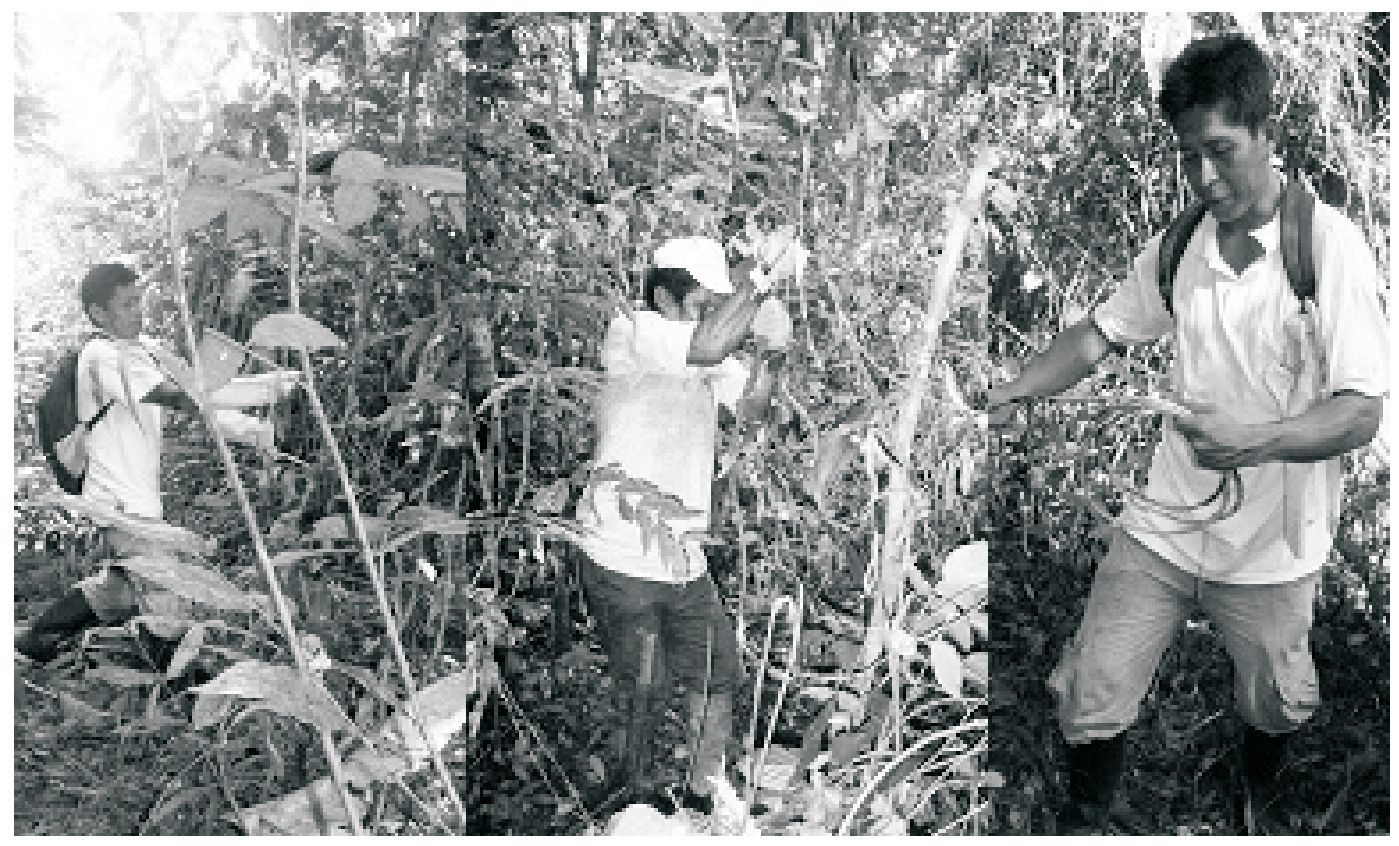

Figura 1. Proceso de extracción de los estípites aprovechables de cashavara Desmoncus polyacanthos sin manejo de los estípites remanentes 


\section{MATERIAL Y MÉTODOS}

\section{ÁREA DEL ESTUDIO}

El estudio se llevó a cabo en los bosques estacionalmente inundables, ubicados en la zona de los lagos Supay y Vainilla (distrito de Jenaro Herrera, Provincia de Requena, Departamento de Loreto). La zona de Jenaro Herrera presenta un clima húmedo tropical, una temperatura media anual $\operatorname{de} 25.9^{\circ} \mathrm{C}$, con fluctuaciones de $\pm 2^{\circ} \mathrm{C}$, la precipitación media anual es de $2715 \mathrm{~mm}$, con una precipitación media mensual entre 140 y $309 \mathrm{~mm}$. (Kvist \& Nebel, 2001). Jenaro Herrera muestra dos paisajes distintos: La Planicie Aluvial Fluviátil, inundada estacionalmente durante el periodo de creciente del Río Ucayali, y La Planicie Interfluvial o Zona de Altura (tierra firme), no influenciada por las variaciones estacionales del nivel de aguas del Río principal (López - Parodi \& Freitas, 1990).

\section{INVENTARIO DE LAS MATA ADULTAS}

Para la instalación del experimento se realizó inventarios en la zona inundable de Jenaro Herrera, en donde se establecieron cuatro parcelas de $50 \mathrm{~m}$ X 50m divididas en sub parcelas de $10 \mathrm{~m}$ X10m, para tomar los siguientes registros de campo: $\mathrm{N}^{\circ}$ de parcela, $\mathrm{N}^{\circ}$ de sub parcela, $\mathrm{N}^{\circ}$ de macolla, $\mathrm{N}^{\circ}$ de estípites sobre maduros, $\mathrm{N}^{o}$ de estípites aprovechables o maduros y $\mathrm{N}^{\mathrm{o}}$ de estípites verdes (remanentes en una macolla).

\section{EVALUACIÓN CUALITATIVA DE LAS PLANTAS}

Posterior a la instalación del experimento, se registraron datos del crecimiento longitudinal de los estípites verdes o remanentes, así como también de los hijuelos o nuevos estípites, el cual consistió en medir desde la base hasta el ápice de la última hoja, utilizan do para ello una wincha graduada en centímetros.

\section{EVALUACIÓN CUALITATIVA DE LAS PLANTAS}

La escala cualitativa que evaluó el vigor de las plantas es la siguiente:

Bueno: Mediano follaje (3 a 5 yemas) color verde intenso, con presencia de color verde pálido, apariencia saludable de las plantas y sus raíces, (Calificación 1).

Regular: Poco follaje (1 a 2 yemas) color predominante verde amarillento apariencia débil de la planta y sus raíces (Calificación 2).

Muerto: Plantas con estípites y hojas secas (Calificación 0).

\section{DISEÑO EXPERIMENTAL DE COSECHA EN CASHAVARA}

Para el caso de cashavara se utilizó un diseño experimental con bloques completamente al azar con cuatro tratamientos y 8 repeticiones (Tabla 1).

\section{CLASIFICACIÓNDE LOS ESTÍPITES}

Estípite Sobre maduros: Son aquellos estípites que han pasado su condición de aprovechables, muchas de ellas llegan al estado de pudrición, debido a que estas no fueron cosechadas en su momento.

Estípite Maduro: Son aquellas raíces que tienen la condición de ser aprovechadas.

Estípites Remanentes: Son aquellos estípites jóvenes que se dejan para futuras cosechas.

Tabla 1. Tratamientos del diseño experimental completamente al azar aplicado al estudio de la cashavara Desmoncus polyacanthos.

\begin{tabular}{c|l|c}
\hline TRATAMIENTO & \multicolumn{1}{c}{ DEFINICIÓN } & REPETICIONES \\
\hline 1 & $\begin{array}{l}\text { Cosecha de 100\% de estípites sobre maduros y } \\
100 \% \text { de estípites aprovechables }\end{array}$ & 8 \\
\hline 2 & $\begin{array}{l}\text { Cosecha de 100\% de estípites sobre maduros, } \\
100 \% \text { de estípites aprovechables y 10\% de estípites remanentes. }\end{array}$ & 8 \\
& $\begin{array}{l}\text { Cosecha de 100\% de estípites sobre maduros, } \\
100 \% \text { de estípites aprovechables y 25\% de estípites remanentes }\end{array}$ & 8 \\
\hline & $\begin{array}{l}\text { Cosecha de 100\% de estípites sobre maduros, } \\
100 \% \text { de estípites aprovechables y 50\% de estípites remanentes }\end{array}$ & 8 \\
\hline
\end{tabular}




\section{RESULTADOS Y DISCUSIONES}

\section{EVALUACIÓN SILVICULTURAL Y MANEJO DE LOS ESTIPITES DE Desmoncus polyacanthos}

En la Tabla 2, se muestra una alta de significancia estadística entre los tratamientos (p-value: $0.001, \mathrm{Ft}$ : $8,42)$, para la variable longitud de estípites $(\mathrm{cm})$; destacándose el tratamiento T3 (manejo de estípites remanentes al $25 \%$ ), con un valor promedio de 883.66 $\mathrm{cm}$. El menor crecimiento longitudinal de estípites lo reportó el T1 (sin manejo de estípites remanentes), con valor promedio de $282,18 \mathrm{~cm}$.

La significancia obtenidas en las medias de los cuatro tratamientos nos permiten afirmar que la especie cashavara en poblaciones naturales o silvestres responden satisfactoriamente al manejo de $25 \%$ de estípites remanentes. Observándose que las plantas de cashavara sin manejo de sus estípites presentaron menor crecimiento debido a la competición de los hijuelos por suelo y luz. Sin embargo en los tratamientos con remoción de hijuelos, la extracción de los estípites atacados por insectos u hongos, estípites totalmente defoliados o rotos, entre otras características de mal estado, permite potencializar la productibilidad de la mata, debido a que seleccionamos los estípites de mejor calidad.

Esto es reforzado por los resultados obtenidos al término de las evaluaciones (Figura 2), donde el mayor promedio está claramente evidenciado en el T3 (manejo del 25\% de los estípites remanentes). Con estos resultados se hace evidente la importancia de la intervención del extractor en el manejo de las macollas al momento de ejecutar la cosecha de los estípites de cashavara, es decir, siempre que el extractor accede al bosque a realizar prácticas de cosecha debe tener en cuenta que se debe cosechar el 100\% de los estípites sobremaduros, $100 \%$ de los estípites aprovechables y $25 \%$ de estípites remanentes que corresponden a estípites sin futuro valor comercial.

\section{EVALUACIÓN DEL VIGOR DE LA CASHAVARA SOMETIDAS A DIFERENTES INTENSIDADES DE COSECHA}

Después de diez meses de evaluación, el 90.6\% de las plantas sometidas a diferentes intensidades de cosecha mostraron un buen vigor y solamente un $3.1 \%$ de plantas murieron cuyo efecto es considerado como baja intensidad para los fines de la investigación (Tabla 3).

Para un análisis más amplio de esta fuente de variación la Figura 3, muestra el efecto del vigor entre los tratamientos, observándose que el Tratamiento tres, T3 (con manejo del 25\% de estípites remanentes) mostró la mejor performance al obtener $100 \%$ de sobrevivencia; manifestándose una vez más las mejores condiciones de manejo con este tratamiento, por otro lado el Tratamiento uno T1 (sin manejo de estípites remanentes) reporta el $13 \%$ de mortandad, siendo la única fuente de variación de los datos en el ensayo, la mortandad de las plantas se deben a una fuerte intensidad de cosecha de los estípites. Además existe diferencia significativa respecto en el Tratamiento tres $\mathrm{T} 3$, respecto a los demás tratamientos, porque asegura una sobrevivencia mínima de hasta el $75 \%$ de las matas bajo estas condiciones de cosecha.

Tabla 2. Prueba de Tukey de tratamientos para el crecimiento Longitudinal de Rá́z (cm) de Cashavara Desmoncus polyacanthos.

\begin{tabular}{|c|c|c|c|c|}
\hline \multicolumn{5}{|c|}{ Test: Tukey Alfa $=0.05 \mathrm{DMS}=333.33997$} \\
\hline \multicolumn{5}{|c|}{ Error: $56743.1156 \mathrm{gl:} 20$} \\
\hline $\mathbf{O M}$ & CLAVE & DESCRIPCIÓN & MEDIAS & SIG. \\
\hline 1 & T3 & Manejo al $25 \%$ de estípites remanentes & 883.66 & a \\
\hline 2 & $\mathrm{~T} 2$ & Manejo al $10 \%$ de estípites remanentes & 540.09 & $b$ \\
\hline 3 & T4 & Manejo al $50 \%$ de estípites remanentes & 321.69 & b \\
\hline 4 & $\mathrm{~T} 1$ & Sin manejo de estípites remanentes & 282.18 & $\mathrm{~b}$ \\
\hline
\end{tabular}

Letras distintas indican diferencias significativas $(p<=0.05)$ 


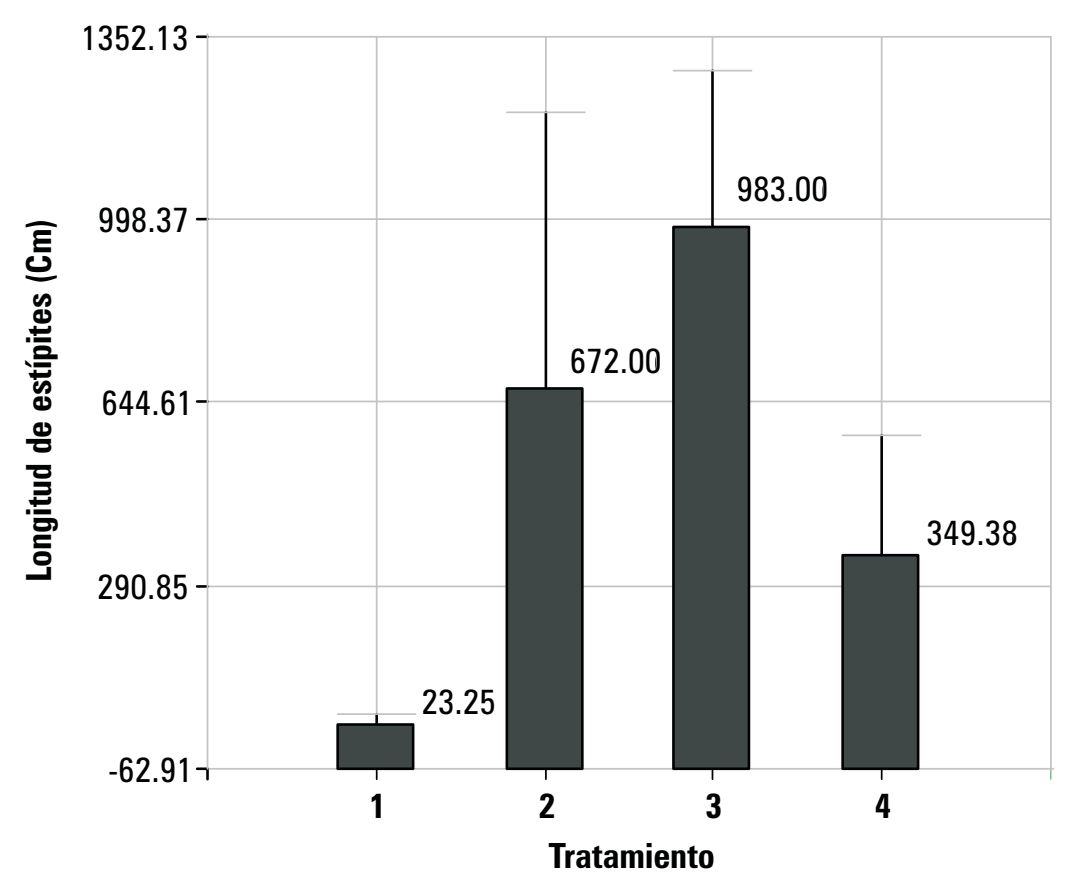

Figura 2. Promedio del crecimiento longitudinal de los estípites $(\mathrm{cm})$ por tratamiento en cashavara Desmoncus polyacanthos.

Tabla 3. Vigor de los estipites de cashavara Desmoncus polyacanthos analizados.

\begin{tabular}{cl|c|c|c|c}
\hline & FRECUENCIA & PORCENTAJE & $\begin{array}{c}\text { PORCENTAJE } \\
\text { VÁLIDO }\end{array}$ & $\begin{array}{c}\text { PORCENTAJE } \\
\text { ACUMULADO }\end{array}$ \\
\hline Válidos & Muerto & 1 & 3.1 & 3.1 & 3.1 \\
& Bueno & 29 & 90.6 & 90.6 & 93.8 \\
& Regular & 2 & 6.3 & 6.3 & 100.0 \\
& Total & 32 & 100.0 & 100.0 & \\
\hline
\end{tabular}


\begin{tabular}{l|l} 
FOLIA & COSECHA DE CASHAVARA Desmoncus polyacanthos MARTIUS, \\
Amazónico & EN LOS BOSQUES INUNDABLES DE LA AMAZZONIA PERUANA
\end{tabular}

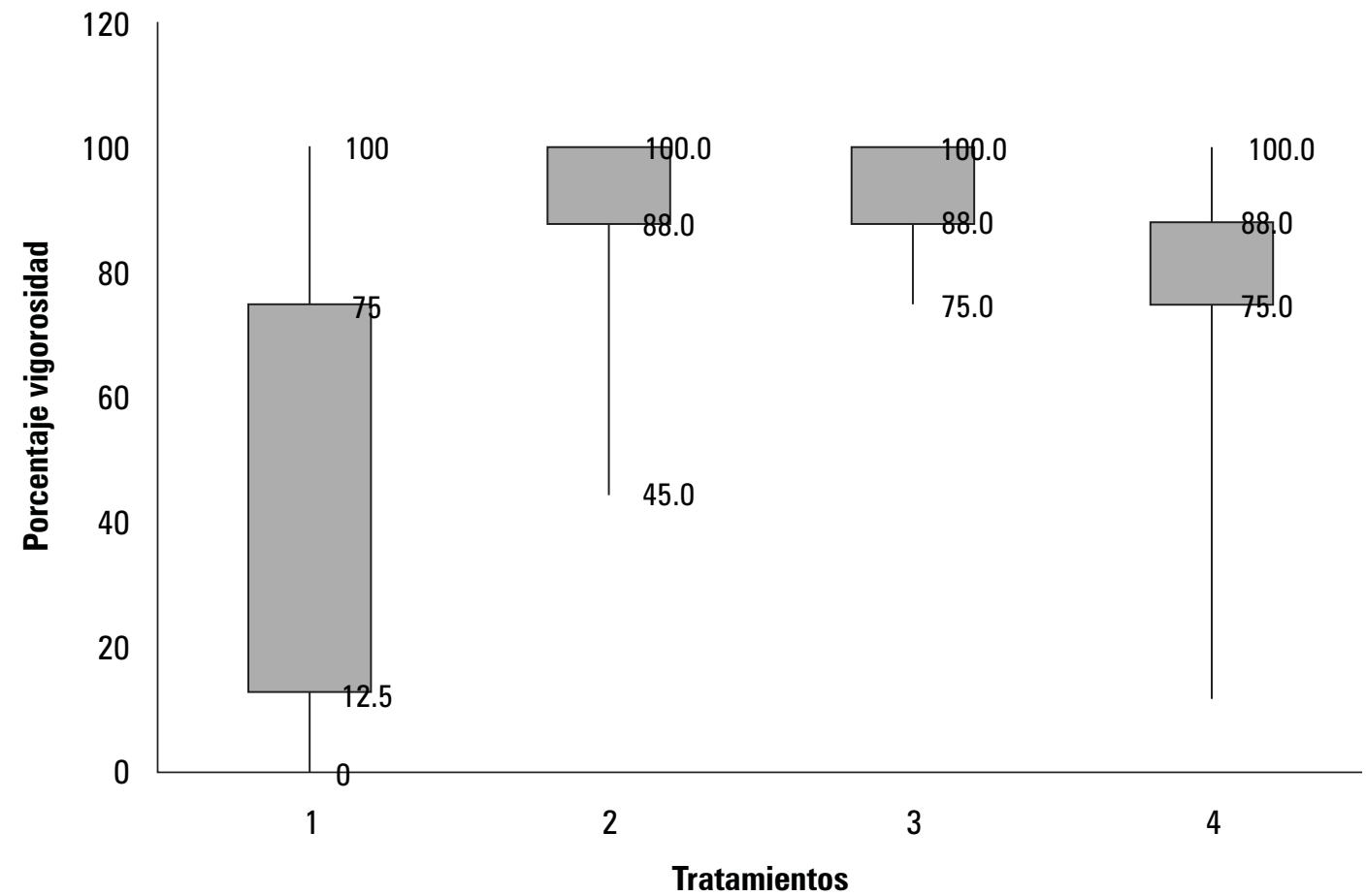

Figura 3. Evaluación del vigor en cashavara Desmoncus polyacanthos en los respectivos tratamientos.

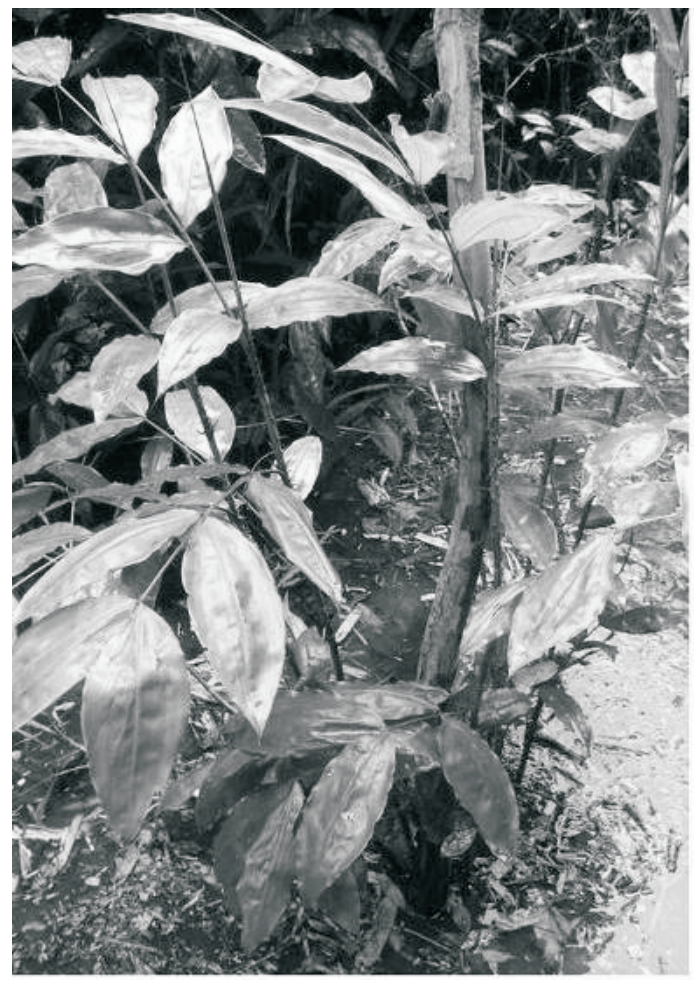

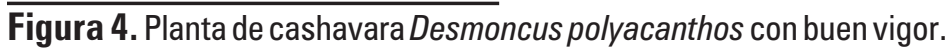




\section{CONCLUSIONES}

Se encontró una alta significancia estadística para los tratamientos; especialmente en el crecimiento longitudinal $(\mathrm{cm})$ de los estípites de cashavara; destacándose nítidamente el tratamiento 3 (manejo de estípites remanentes al 25\%), con un valor promedio de $883.66 \mathrm{~cm}$. El menor crecimiento longitudinal $(\mathrm{cm})$ de estípites lo reportó el T1 (sin manejo de los estípites remanentes), con valor promedio de $282.18 \mathrm{~cm}$. Lo que nos permite estimar que la especie responde adecuadamente al manejo de los estípites remanentes, con una cosecha controlada dejando un $75 \%$ de estos estípites inalterados; extrayendo el $25 \%$ de los estípites remanentes sin futuro valor comercial.

Las evaluaciones del vigor de D. polyacanthos en poblaciones naturales, sometidas a diferentes tratamientos e intensidades de cosecha, reporta un $90.6 \%$ de plantas que mostraron buen vigor, el 6.3\%, con regular vigor y un $3.1 \%$ de plantas murieron cuyo efecto es considerado como de baja intensidad para los fines de la investigación.

Mediante el análisis de los resultados se demostró que en todos los parámetros de evaluación el Tratamiento tres (T3) que consiste en cosechar el 100\% de los estípites sobre maduros, $100 \%$ de los estípites aprovechables y $25 \%$ de estípites remanentes fue significativamente mejor a los demás tratamientos, por consiguiente correspondería a escogerse como una técnica para el aprovechamiento sostenible de cashavara en los bosques inundables de la Amazonía Peruana.

\section{AGRADECIMIENTO}

Al Proyecto de Investigación y Extensión Agrícola para la Innovación y Competitividad del Agro Peruano (PIEA-INCAGRO) del Ministerio de Agricultura por el financiamiento del presente trabajo de investigación; a los señores: Carlos Shapiama, Geiner Murayari, Angel Jiménez, Juan Sánchez y Julio Irarica por el apoyo en las evaluaciones de campo.

\section{BIBLIOGRAFÍA CITADA}

Chinchilla, M. 1993. Caracterización preliminar del bayal (Desmoncus spp) en Aldea La Pasadita, San Andrés Petén. Universidad de San Carlos de Guatemala, Facultad de Agronomía. Proyecto OLAFO. 46p.

Henderson, A. Galeano, G.; Bernal, R. 1995. Field Guide to the palms of the Americas. Princeton. University Press. Princeton, New Jersey. 199p.

Kvist, L. P.; Nebel, G. 2001. A review of Peruvian flood plain forests: ecosystems, inhabitants and resource use. In: Forest Ecology Management (150)3-26.

López, J. P.; Freitas, D. 1990. Geographical aspects of forested wetlands in the lower Ucayali. Peruvian. For. Ecol. Manageme 33/34. 157-158p.

Muños, F.; Carpio, I. 1995. Uso potencial de la palma de Bayal como sustituto del Ratán. Universidad de Costa Rica. Proyecto OLAFO - CATIE. 24p.

Pineda, P.; Marmillod, D.; Ferreira, P.; Ocampo, R. 1995. Elementos de muestreo para el diseño de inventario de bayal (Desmoncus sp) en el bosque petenero. In: Centro agronómico Tropical de Investigación y Enseñanza. Segunda semana científica 1994 - 1995; resúmenes. Turrialba. Costa Rica. pp 103-107.

Torres, G.; Delgado, C.; MejiaA, K. 2010. Potential Harvest of Desmoncus polyacanthos (Arecaceae) in the Peruvian Amazonia. Revista Palms. Journal of the international Palm Society. Vol 54(2) 73-76. Jun 2010. 


